\section{Benefícios da amamentação para a saúde da mulher e da criança: um ensaio sobre as evidências}

\author{
Benefits of breastfeeding for maternal and child \\ health: an essay on the scientific evidence
}

\footnotetext{
${ }^{1}$ Instituto de Saúde, Secretaria de Estado da Saúde de São Paulo, São Paulo, Brasil.

Correspondência T. S. Toma Instituto de Saúde, Secretaria de Estado da Saúde de São Paulo.

Rua Santo Antonio 590, São Paulo, SP 01314-000, Brasil. ttoma@isaude.sp.gov.br
}

\begin{abstract}
This paper provides a literature review on breastfeeding, selecting studies that have helped explain its benefits for maternal and child health. A search for articles published since 2000 was conducted, including relevant studies for the advancement of knowledge in previous decades. An Internet search of the PubMed and SciELO databases was performed to select the studies. Besides the aspects on which there is widespread agreement, controversial results were also included, as well as intriguing ones from the field of neurobiology. Public policy recommendations have undergone substantial changes following those new discoveries. Some studies have also been conducted to search for the most cost-effective measures to promote breastfeeding practices. This paper highlights: current recommendations on child feeding; the importance of breastfeeding for early childhood; the implications of breastfeeding for health of both the infant and mother; and the effectiveness of key interventions to encourage breastfeeding.
\end{abstract}

Nutritional Epidemiology; Breast Feeding; Human Milk; Women's Health; Child's Health (Public Health)
Tereza Setsuko Toma ${ }^{1}$

Marina Ferreira Rea 1

\section{Introdução}

Pesquisas realizadas nas duas últimas décadas contribuíram muito para uma melhor compreensão dos benefícios do aleitamento materno para a criança e para a mulher. A relevância dos achados levou a mudanças substanciais nas recomendações para políticas públicas. Muitos estudos também têm sido realizados com o objetivo de avaliar quais intervenções seriam mais efetivas para um aumento das práticas de amamentação. Este ensaio procurou destacar alguns aspectos dos estudos sobre amamentação publicados desde o ano 2000, particularmente revisões sistemáticas. Muitos desses estudos apresentam resultados de projetos recentes nos quais se procurou utilizar coleta de dados e indicadores comparáveis de aleitamento.

O texto procura abordar: (1) As Recomendações sobre Alimentação de Crianças Pequenas; (2) A Importância da Amamentação no Início da Vida; (3) As Implicações do Aleitamento Materno para a Saúde da Criança; (4) As Implicações do Aleitamento Materno para a Saúde da Mulher; e (5) A Efetividade de Algumas Ações Pró-amamentação.

\section{As recomendações sobre alimentação de crianças pequenas}

Em meados da década de 1980, publicam-se pela primeira vez estudos que comprovam a im- 
portância de amamentar exclusivamente, sem qualquer outro líquido, água ou chá, levando à menor risco de morbidade 1 e mortalidade 2 . Esses estudos, sobejamente conhecidos, assim como outros realizados em diversos países, forneceram novas bases para a reformulação de políticas internacionais, particularmente da Organização Mundial da Saúde (OMS) e do Fundo das Nações Unidas para a Infância (UNICEF). Essas novas diretrizes recomendam que as crianças sejam amamentadas de forma exclusiva até os seis meses e, que após este período, gradativamente se inicie a alimentação complementar mantendo a amamentação até pelo menos os dois anos de idade 3 .

Assim, inicia-se a busca junto a renomados cientistas e profissionais de saúde pública com o objetivo de reunir idéias e ações para compor políticas e programas que possam levar ao aumento da prática do aleitamento materno exclusivo nos primeiros meses de vida. Propõem-se também consultas técnicas sobre alimentação complementar concomitante com a amamentação, integrando-se propostas de capacitação profissional que, já neste século, passam a cobrir o período do nascimento até o segundo ano de vida. Mais ainda, incorporam-se nos anos recentes as propostas de ações nas emergências, endemias e epidemias (como a AIDS), eventos que certamente interferem nas decisões sobre alimentação da criança pequena 3 .

Nos anos 90, analisando-se os dados do Demographic and Health Surveys (DHS) para diversos países, observa-se que o aleitamento materno exclusivo até quatro meses cresceu de $46 \%$ para $53 \%$. E quando se considera aleitamento materno exclusivo até seis meses, esse crescimento foi de $34 \%$ para $39 \%$. Isso se deveu principalmente à implementação da Iniciativa Hospital Amigo da Criança e do Código Internacional de Comercialização de Substitutos do Leite Materno 4 . Depois do ano 2000 espera-se que a Estratégia Global sobre Alimentação de Lactentes e Crianças de Primeira Infância 3 seja a política pública a levar a um maior aumento dessas práticas.

Amamentar exclusivamente até o sexto mês de vida tornou-se recomendação baseada em revisão extensa da literatura, solicitada pela OMS a partir de duas buscas independentes ${ }^{5}$. Estas incluíram os bancos de dados: MEDLINE (desde 1966), Index Medicus (antes de 1966), CINAHL, HealthSTAR, BIOSIS, CAB Abstracts, EMBASEMedicine, EMBASE-Psychology, Econlit, Index Medicus para a Região da OMS Eastern Mediterranean, African Index Medicus, LILACS, EBM Reviews-Best Evidence, Revisões sistemáticas do Cochrane Database e Cochrane Controlled Trials Register. As buscas levaram a 2.668 trabalhos publicados sobre o tema. Vinte estudos foram selecionados: 9 de países em desenvolvimento (dois dos quais de Honduras, experimentos controlados) e 11 de países desenvolvidos (todos observacionais). Nenhum deles mostrou que amamentar por seis meses pode comprometer o ganho de peso ou altura. Bebês amamentados exclusivamente apresentaram menor morbidade por diarréia em comparação com aqueles que receberam aleitamento materno junto com alimentos complementares aos 3-4 meses. Os resultados foram conflitantes quanto a ferro e anemia, pois nos países em desenvolvimento onde os estoques de ferro no recém-nascido costumam ser baixos, o aleitamento materno exclusivo sem suplementação com ferro pode comprometer a situação hematológica destas crianças. Não houve redução significativa de risco de eczema, asma e outras atopias. Aleitamento materno exclusivo mostrou-se também importante para a saúde da mulher: quando praticado por seis meses esteve associado a retardo na volta da menstruação e maior rapidez na perda de peso pós-parto 5 .

Por muito tempo persistiam críticas sobre provável crescimento mais lento de crianças amamentadas quando comparadas às alimentadas com fórmula infantil, usando-se o referencial do Centro Nacional para Estatísticas em Saúde dos Estados Unidos, conhecido como curvas NCHS. O reconhecimento das limitações dessa referência internacional desencadeou a construção de novos padrões ${ }^{6}$. A OMS lançou, em 2006, as curvas de crescimento elaboradas com base em uma amostra de crianças vivendo em condições que permitiam o alcance de seu potencial genético de crescimento. Essas curvas, mais do que uma referência, são consideradas um padrão por serem o resultado de um estudo multicêntrico do qual participaram Brasil, Gana, Índia, Noruega, Omã e Estados Unidos, e terem sido incluídas apenas crianças amamentadas de famílias em boa situação econômica, filhas de mulheres não-fumantes 7. Os resultados mostram a similaridade de crescimento de crianças desses diferentes países quando dadas as condições adequadas para o seu desenvolvimento. Entretanto, por se tratar de um novo parâmetro há preocupação quanto às implicações práticas, ainda não totalmente avaliadas, de sua adoção em larga escala pelas agências e países 8 .

Em que pese a grande insistência que se tem sobre o não uso de outros fluidos que não o leite materno nos primeiros seis meses, um estudo de quase 10 mil crianças e mães em Gana, Índia e Peru mostrou que bebês predominantemente amamentados foram também protegidos quanto à mortalidade, apresentando riscos de morte similares aos exclusivamente amamentados 9; 
no entanto, bebês não amamentados tiveram um alto risco de morte quando comparados aos predominantemente amamentados, ou mesmo parcialmente amamentados. Os autores sugerem que em locais onde a amamentação predominante é prevalente (amamentação mais uso de água ou chá nos primeiros seis meses de vida) os esforços de promoção devem se concentrar em sustentar essas práticas em vez de tentar insistir apenas no aleitamento materno exclusivo 9 .

Após os seis meses, iniciar o consumo de alimentos complementares é recomendável para que todas as necessidades nutricionais de uma criança em franco crescimento sejam adequadamente atendidas. Contudo, manter a amamentação também é importante porque o aporte de $500 \mathrm{ml}$ diários de leite materno ainda será capaz de fornecer cerca de $75 \%$ das necessidades de energia, $50 \%$ das de proteína e $95 \%$ das de vitamina A, além da proteção imunológica 10 .

Em março de 2003, publicou-se um número especial do Food and Nutrition Bulletin com base nos resultados da consulta técnica sobre alimentação complementar, organizada pela OMS. Essa revista traz importantes contribuições referentes à atualização de aspectos técnicos, promoção e apoio para melhorar as práticas alimentares, formas de melhorar a disponibilidade de alimentos e de lidar com as tecnologias no âmbito domiciliar. Em um de seus artigos 11, discute-se que as publicações sobre alimentação infantil têm enfatizado a importância de continuar a amamentação após o início da alimentação complementar, porém não costumam discutir como as mães podem manter uma boa produção de leite. Sabe-se que as crianças têm capacidade de auto-regular a ingestão de calorias de acordo com suas necessidades, portanto, se receberem a energia necessária de outros alimentos diminuirão a ingestão de leite materno. Nesse sentido, segundo esses autores, é plausível pensar que o grau de substituição do leite materno seja influenciado por fatores como a freqüência e a densidade energética dos alimentos, o horário das mamadas e o modo como são oferecidos os alimentos. Quais seriam, então, as possíveis recomendações úteis para uma alimentação apropriada nessa fase da vida? Frente à escassez de dados de pesquisa e a dificuldade de estabelecer qual o número adequado de mamadas em diferentes faixas de idade, seria importante recomendar que a mãe amamentasse de acordo com o desejo da criança. Sobre o uso de mamadeiras, além de maior risco de contaminação, de longa data acredita-se que estes artefatos têm mais chances de substituir a amamentação do que quando se alimenta com copo ou colher. Nesse sentido, outra recomendação seria evitar o uso de mamadeiras. Uma questão sensível é ensinar o cuidador a observar e responder aos sinais de fome e saciedade da criança (responsiveness), alimentando-a até que recuse o alimento e sem forçá-la a comer. E nos casos em que a grande preferência da criança pela amamentação prejudica o consumo de alimentos complementares seria recomendável que estes fossem dados antes das mamadas 11 .

\section{A importância da amamentação no início da vida}

Em anos recentes põe-se novamente em destaque a importância de se iniciar precocemente a amamentação. Um estudo realizado em quatro distritos rurais de Gana analisou dados de 11.316 crianças não gêmeas nascidas entre julho de 2003 e junho de 2004, sobreviventes ao segundo dia após o nascimento e que haviam iniciado a amamentação 12. Os principais achados apontam que a amamentação precoce pode levar a uma considerável redução na mortalidade neonatal. Essa mortalidade por todas as causas poderia ser reduzida em $16,3 \%$ se todas as crianças iniciassem a amamentação no primeiro dia de vida, e em $22,3 \%$ se a amamentação ocorresse na primeira hora. Os efeitos da amamentação sobre a redução da mortalidade infantil já são conhecidos há alguns anos, como citado acima, mas este parece ter sido o trabalho pioneiro a discutir a importância da amamentação precoce na prevenção da mortalidade neonatal. Os autores procuram justificar como a amamentação precoce poderia afetar o risco de morrer no período neonatal. Os mecanismos seriam pelo menos os seguintes: (1) as mães que amamentam logo após o parto têm maior chance de serem bem sucedidas na prática da amamentação; (2) os alimentos pré-lácteos, comumente oferecidos aos bebês antes da amamentação, podem ocasionar lesões no intestino imaturo; (3) o colostro acelera a maturação do epitélio intestinal e protege contra agentes patogênicos; (4) o contato pele a pele previne a ocorrência de hipotermia ${ }^{12}$. Alguns desses aspectos são discutidos a seguir com maior aprofundamento.

A preocupação com a hipoglicemia neonatal logo após o parto tem sido responsável por interrupção do aleitamento materno exclusivo em muitos hospitais devido ao uso de soro glicosado em recém-nascidos. No entanto, a hipoglicemia parece ser comum entre os mamíferos como um processo adaptativo e autolimitado, na medida em que os níveis de glicose acabam por aumentar espontaneamente em poucas horas. A amamentação precoce e exclusiva atende às necessidades dos recém-nascidos a termo, por isto desaconse- 
lha-se o uso de soro glicosado, água ou fórmula infantil que podem interferir no estabelecimento da amamentação e nos mecanismos metabólicos compensatórios do bebê. Recomenda-se que o monitoramento dos níveis glicêmicos seja realizado apenas nos recém-nascidos de risco ou com sintomas clínicos de hipoglicemia, uma vez que os exames de rotina em bebês saudáveis podem interferir negativamente na relação mãe/bebê e na amamentação 13 .

Crianças nascidas de parto normal domiciliar e amamentadas exclusivamente apresentam uma flora intestinal benéfica, com maior quantidade de bifidobactérias e menos Clostridium dificile e Escherichia coli 14. Segundo esse estudo, que examinou as fezes de 1.032 bebês holandeses de até um mês de idade, os principais fatores determinantes da microflora intestinal no início da vida são o tipo de parto, a alimentação infantil, a idade gestacional, a hospitalização e o uso de antibióticos. Já em 1905, havia registros de diferenças na composição da microflora intestinal de crianças amamentadas em comparação a crianças desmamadas. A partir da descoberta do fator bifidus, na década de 1970, torna-se cada vez mais conhecido o mecanismo pelo qual ocorre a proteção da mucosa intestinal contra os agentes patogênicos. Sabe-se hoje que vários tipos de oligossacarídeos e glicoconjugados presentes no leite materno, conhecidos como agentes pré-bióticos, estimulam a colonização do intestino por microorganismos benéficos. Esses agentes atuam na primeira etapa essencial da patogênese ao impedir que um microorganismo se fixe na parede celular 15 .

Algumas práticas durante o trabalho de parto e logo após o nascimento podem facilitar o início da amamentação. Estudo recente chama a atenção de que entre elas estão a implementação da Iniciativa Hospital Amigo da Criança (que veremos adiante), o não uso de sedativo particularmente próximo ao final do primeiro estágio do trabalho de parto, a não separação entre mãe e bebê, e o contato pele a pele logo após o parto 16 . Nesta revisão, as autoras discutem também a importância de proporcionar cuidados apropriados à mulher, uma vez que experiências estressantes de parto estão associadas à menor sucesso na amamentação e retardo no início do processo de lactação.

Uma revisão sistemática sobre o contato precoce pele a pele entre mães e seus recém-nascidos encontrou efeitos positivos sobre a primeira mamada, amamentação de um a quatro meses pós-parto, duração da amamentação, ingurgitamento mamário e reconhecimento do odor do leite materno pelo bebê 17 . Nenhum efeito negativo foi identificado. $\mathrm{O}$ contato precoce entre mãe e recém-nascido, entretanto, parece não receber ainda a devida atenção por parte dos profissionais de saúde responsáveis pela condução da grande maioria dos partos e nascimentos nos dias atuais.

Mercer et al. 18 realizaram um levantamento das evidências científicas a respeito dos procedimentos comumente utilizados na atenção ao recém-nascido. A conclusão é que muitas dessas práticas não têm uma eficácia comprovada e deveriam ser modificadas por interferirem negativamente na relação mãe/bebê. O clampeamento do cordão, de acordo as evidências disponíveis, deveria ser postergado como medida de prevenção da anemia na infância. Os estudos não demonstram benefícios das aspirações rotineiras da boca e nariz ao nascer e estas deveriam ser abolidas em recém-nascidos normais. A aspiração gástrica mostrou ser danosa e não deveria ser utilizada como cuidado de rotina. Até mesmo na manobra de ressuscitação, a tendência é recomendar o uso do ar ambiente em primeiro lugar, deixando o oxigênio a $100 \%$ para os casos de não resposta. O contato pele a pele, por sua vez, apresenta-se como um procedimento seguro, barato e apropriado para regulação da temperatura corporal do recém-nascido sadio, além de apresentar benefícios de curto e longo prazos para mães e crianças 18 .

O contato pele a pele desencadeia uma série de eventos hormonais importantes para a relação mãe/bebê. O toque, o odor e o calor estimulam o nervo vago e isto, por sua vez, faz com que a mãe libere ocitocina, hormônio responsável, entre outras ações, pela saída e ejeção do leite. Esse hormônio faz com que a temperatura das mamas aumente e aqueça o bebê. Por outro lado, a ocitocina reduz a ansiedade materna, aumenta sua tranqüilidade e responsividade social 18,19.

Um aspecto menos destacado nos estudos sobre amamentação é quanto a sua prática pode facilitar o desenvolvimento de um forte apego ao cuidador, aspecto fundamental para a sobrevivência daquelas espécies cujo desenvolvimento ocorre em grande parte fora do útero. Os aspectos comportamentais do apego têm sido bastante estudados e, mais recentemente, surgem dados também sobre sua neurobiologia. Embora provenientes de estudos com animais, os achados têm sido úteis para a compreensão cada vez maior da importância desses fenômenos entre os humanos. Em 2005, dois estudiosos avaliaram o circuito neural que possibilita um rápido apego dos filhotes de ratos ao seu cuidador, ação necessária para sua sobrevivência no ninho 20 . Os achados sugerem que o cérebro neonatal não é uma versão imatura do cérebro adulto; pelo contrário, ele parece ter sido dese- 
nhado de uma maneira única para aperfeiçoar o apego ao cuidador.

Outro estudo em que foram acompanhados 174 pares mãe/bebê durante o primeiro ano após o nascimento, concluiu que o fator preditivo mais importante para um apego seguro é a qualidade da interação diádica na infância 21 . A amamentação não apresentou uma relação direta com o apego seguro, entretanto os dados sugerem que as mães que optam por amamentar aparentam ser mais responsivas aos sinais de suas crianças durante o processo de interação no início da vida. Então, indiretamente a amamentação contribuiria para fomentar um apego seguro. Uma criança que tenha uma representação mental de pais responsivos e disponíveis tem maior probabilidade de apresentar um apego seguro, ao passo que o apego inseguro ocorre quando falta este tipo de representação. A importância disso é que os pais, para uma criança adequadamente apegada, representam uma base segura a partir da qual poderá explorar o ambiente. A sensibilidade materna também se mostrou associada com a intenção e a duração pretendida de amamentar durante o período pré-natal, dado sugestivo de que esta intenção poderia ser um marcador precoce da posterior sensibilidade da mãe com relação ao bebê 21 .

A teoria do apego, cujo modelo teórico foi desenvolvido por Bowlby 22 na década de 1960, tem sido utilizada até hoje nos estudos avaliativos neste campo. Sua teoria foi considerada revolucionária por introduzir na discussão conceitos relativos à adaptabilidade evolutiva do homem. O apego, que Bowlby 22 diferencia de vínculo, implica um comportamento no qual a criança busca manter a proximidade com uma figura específica e seu cerne é o estabelecimento do senso de segurança. As bases teóricas elaboradas por Bowlby receberam grande contribuição das observações de crianças da tribo Ganda realizadas por Ainsworth et al. (1978, apud Brum \& Schermann 23). A qualidade das relações de apego, classificada por Ainsworth em três categorias, seria dependente das interações entre cuidador e criança: (1) bebês apegados de maneira segura à mãe; (2) bebês esquivos e apegados de maneira ansiosa e (3) bebês resistentes e apegados de maneira ansiosa 22,23.

Estudos como esses têm contribuído para o quadro das evidências científicas que reforçam as recomendações feitas em 1989 pela OMS e UNICEF, posteriormente incorporadas pela já mencionada Iniciativa Hospital Amigo da Criança 10 , que sintetiza dez passos para o sucesso do aleitamento materno, entre os quais: iniciar a amamentação na primeira hora de vida (passo
4), não dar outros líquidos além do leite materno a recém-nascidos (passo 6) e não separar mães e bebês desnecessariamente (passo 7).

\section{As implicações do aleitamento materno para a saúde da criança}

Estimativas recentes quanto a diversas formas de ação e suas conseqüências para a saúde da criança mostraram que a promoção do aleitamento materno exclusivo é a intervenção isolada em saúde pública com o maior potencial para a diminuição da mortalidade na infância.

Interessante discussão sobre a questão da sobrevivência infantil no mundo e, em particular, nas Américas mostra que existe uma impressão equivocada acerca do controle da mortalidade infantil e a escassez de recursos 24 . Recursos têm sido direcionados para outros problemas de saúde relevantes, o que é apontado como possível justificativa para compreender a diminuição nos investimentos financeiros em saúde da criança. No entanto, a cada ano ainda morrem mais de dez milhões de crianças menores de cinco anos no mundo e parece difícil atingir a quarta das oito Metas do Milênio das Nações Unidas, que é reduzir em dois terços a mortalidade de crianças abaixo de cinco anos de 1990 a 2015, embora o Brasil esteja bem colocado entre os países a atingir tal meta. Esse fato levou à formação do Grupo de Estudos da Sobrevivência Infantil de Bellagio, que publicou uma série de cinco artigos no Lancet em 2003.

Nas Américas, Brasil e México estão entre os 42 países onde ocorre a maioria dessas mortes. A amamentação e a alimentação complementar estão incluídas entre as 23 intervenções viáveis, efetivas e de baixo custo identificadas por esse grupo de estudiosos 24 .

Em 2005, o Lancet publica outra série de artigos com foco sobre sobrevivência neonatal 24 . Discute-se que, embora as mortes no período neonatal sejam o principal componente da mortalidade hoje em dia, isto tem sido ignorado pelas agências financiadoras. Nessa série, estimase que $38 \%$ das mortes de crianças menores de cinco anos ocorram nas primeiras quatro semanas de vida, para as quais foram identificadas 16 possíveis intervenções. A amamentação foi considerada uma intervenção para a qual existem evidências sobre eficácia incontestável e que, com base em estudos de efetividade, mostrou ser viável para implementação em larga escala 25 .

Com relação às crianças brasileiras, os dados mostram uma clara desvantagem das crianças pobres quanto à cobertura das intervenções de sobrevivência infantil. Além disso, os autores dis- 
cutem que não basta alcançar uma boa cobertura, uma vez que para serem efetivas as intervenções precisam ser de boa qualidade 24 .

Há evidências de que, tanto em países em desenvolvimento quanto nos desenvolvidos, a amamentação protege as crianças contra infecções dos tratos gastrintestinal e respiratório, sendo maior a proteção quando a criança é amamentada de forma exclusiva e por tempo prolongado 26 .

Na década de 80, Feachem \& Koblinsky 27 constataram por meio da revisão de 35 estudos que a amamentação estava relacionada a um menor risco de morbidade e mortalidade por diarréia. Os estudos apresentavam muitos problemas metodológicos e mais pesquisas seriam necessárias para esclarecer os aspectos pouco compreendidos da relação entre amamentação e diarréia. No entanto, afirmavam os autores, isso não deveria servir de motivo para retardar as ações de promoção do aleitamento materno. $\mathrm{Na}$ linha dos estudos metodologicamente bem conduzidos, o de Victora et al. 2 trouxe grande contribuição ao mostrar que o tipo de leite da dieta infantil tinha grande influência sobre os riscos de morte por diarréia e doenças respiratórias. Crianças amamentadas que não recebiam outro leite além do materno, ao serem comparadas com crianças desmamadas, apresentaram risco 14 vezes menor de morrer por diarréia no primeiro ano de vida. Ao se estudar apenas as crianças menores de dois meses de idade, o risco entre as desmamadas era 23 vezes maior quando comparadas às amamentadas. Huffman \& Combest 28 citam outro estudo realizado no Peru, em que crianças amamentadas exclusivamente apresentavam um menor risco de adoecer por diarréia em comparação a crianças amamentadas e que tomavam água. Daí os autores recomendarem que a amamentação exclusiva deveria ser estendida por pelo menos 4 a 6 meses.

Os efeitos protetores da amamentação contra infecções do ouvido e pulmão têm-se tornado mais evidentes nos últimos anos. Nesse particular, cumpre importante papel a imunoglobulina A (IgA) secretora, um anticorpo resultante da resposta da mãe à exposição prévia a agentes infecciosos. Ela tem como característica sobreviver nas membranas das mucosas respiratória e gastrintestinal e ser resistente à digestão proteolítica. Além de impedir que agentes patogênicos se fixem nas células da criança amamentada, ela limita os efeitos danosos do processo inflamatório 29.

A amamentação exclusiva protege as crianças pequenas de evoluírem para quadros mais graves de infecção respiratória. Estudo de caso-controle aninhado realizado em Pelotas, Rio
Grande do Sul, Brasil, analisou as internações por pneumonia no período pós-neonatal de uma coorte de 5.304 crianças. Crianças não amamentadas apresentaram risco 17 vezes maior de serem internadas por pneumonia do que as que recebiam apenas leite materno. A não-amamentação afetou ainda mais as crianças abaixo de três meses de idade, cujo risco relativo para internação por pneumonia foi de $61 \% 30$. A amamentação predominante por pelo menos seis meses e a amamentação parcial até um ano de idade também podem reduzir a prevalência de infecções respiratórias na infância. Estudo de uma coorte prospectiva de 2.602 crianças australianas desde o nascimento, analisou a relação entre duração da amamentação e doenças respiratórias e infecções durante o primeiro ano de vida. Os autores relatam que a amamentação predominante por pelo menos seis meses mostrou ser fator protetor significativo, reduzindo a freqüência de consultas médicas e internações, particularmente por infecções respiratórias do trato superior e chiado. Interromper a amamentação antes dos 12 meses mostrou ser fator de risco para consultas médicas por doença respiratória 31 .

Sabe-se que a bronquiolite é importante causa de morbidade em crianças pequenas. Em estudo realizado na Grécia com 240 crianças abaixo de dois anos internadas por bronquiolite, observou-se que a amamentação por mais de quatro meses melhora o quadro de gravidade mesmo em crianças de famílias de fumantes 32 .

Quanto à morbidade por algumas causas específicas, observam-se controvérsias: quanto à asma um estudo mostrou que amamentar protegeu as crianças oriundas de 221 famílias com história de atopia comparadas com 308 de famílias com história negativa 33. Por outro lado, estudo conduzido na Austrália observando uma coorte de 516 crianças aos cinco anos, também oriundas de famílias com história de atopia, não encontrou associação entre tempo prolongado de amamentação e presença de asma, eczema ou atopia 34. Existem também diferenças na presença de atopia por sexo da criança e conforme seu pai ou sua mãe tenham sido portadores de asma ${ }^{35}$. Outro estudo prospectivo de mais de mil crianças belgas seguidas desde a gravidez até os primeiros 12 meses, também mostrou que no primeiro ano de vida a amamentação não tem efeito protetor quanto a eczema 36. Mesmo revisões sistemáticas não conseguem dar uma resposta sobre essa relação "amamentação/atopia”, o que indica que melhores estudos precisam ser elaborados.

Há controvérsia também sobre a alimentação artificial como fator de risco para morte súbita. Ao passo que alguns estudos observaram maior prevalência de alimentação artificial entre os ca- 
sos do que entre os controles, outros não apontaram qualquer diferença. Foi observada uma associação entre amamentação exclusiva e redução da síndrome da morte súbita após controlar para variáveis de confusão, tais como fumo durante a gravidez, emprego paterno, posição ao dormir e idade da criança 37 . O papel da amamentação como mecanismo de proteção contra morte súbita ainda não foi completamente elucidado. Entre as possíveis explicações estão a menor incidência de infecções, as mamadas freqüentes e o contato mais estreito entre mãe e criança.

Quanto à infecção urinária, foi avaliado um possível efeito protetor da amamentação contra o primeiro episódio acompanhado de febre em crianças suecas menores de seis anos. Os 200 casos de infecção urinária foram comparados a 336 controles pareados por idade e gênero. Observou-se um efeito protetor nas crianças que estavam em amamentação exclusiva, assim como uma proteção até os dois anos de idade, mesmo em crianças desmamadas 38 .

Com relação aos efeitos de longo prazo da amamentação, uma revisão sistemática com dados obtidos do MEDLINE (1966 a março de 2006) e do Scientific Citation Index Databases concluiu que as evidências disponíveis sugerem que ela oferece benefícios 39. Os resultados mostraram que crianças amamentadas apresentaram médias mais baixas de pressão sanguínea e de colesterol total, e melhor desempenho em testes de inteligência. As prevalências de sobrepeso/obesidade e diabetes tipo 2 também foram menores.

\section{As implicações do aleitamento materno para a saúde da mulher}

As implicações da amamentação para a saúde da mulher ainda precisam ser mais amplamente estudadas. Diversos trabalhos recentes eliminaram a controvérsia sobre a diminuição do risco de câncer de mama entre as mulheres que amamentaram prolongadamente. Revisão da literatura foi publicada no Brasil 40 e novos estudos têm sido apresentados em países, culturas e etnias diversas.

Sobre a proteção contra câncer de mama, recentemente foram avaliados 256 casos comparados a 536 controles em Israel; os resultados mostraram que mulheres judias com duração mais curta de amamentação, início tardio da primeira mamada e percepção de "leite insuficiente" apresentaram maiores riscos de ter câncer de mama 41. Em outra etnia, a coreana, 753 casos de câncer de mama e igual número de controles foram comparados, observandose um efeito protetor dose-dependente, sendo que 11-12 meses de amamentação reduziram em $54 \%$ o risco, comparado a 1-4 meses 42 . Uma revisão de 47 estudos realizados em 30 países envolvendo cerca de 50 mil mulheres com câncer de mama e 97 mil controles, sugere que o aleitamento materno pode ser responsável por 2/3 da redução estimada no câncer de mama. A amamentação foi tanto mais protetora quanto mais prolongada: o risco relativo de ter câncer decresceu $4,3 \%$ a cada 12 meses de duração da amamentação, independentemente da origem das mulheres (países desenvolvidos versus não desenvolvidos), idade, etnia, presença ou não de menopausa e número de filhos. Estimouse que a incidência de cânceres de mama nos países desenvolvidos seria reduzida a mais da metade (de $6,3 \%$ para $2,7 \%$ ) se as mulheres amamentassem por mais tempo 43.

Por outro lado, ter sido amamentada quando bebê também mostrou relação com a incidência de câncer de mama na idade adulta: foi analisada uma coorte de 4.999 sujeitos iniciada nos anos 30 e realizada uma extensa meta-análise de outros estudos, concluindo-se que ter sido amamentada está relacionado a menor risco de câncer na pré-menopausa 44.

Um estudo caso-controle realizado em hospital japonês envolvendo 155 mulheres com câncer do endométrio e 96 controles encontrou um maior risco deste câncer entre aquelas multíparas que nunca haviam amamentado; os autores referem que o aumento verificado nos casos de câncer de endométrio pode estar relacionado à diminuição da prática de amamentar e da paridade de mulheres do Japão 45 .

Indaga-se sobre o efeito da amamentação no menor risco de morte por artrite reumatóide e há também controvérsia quanto a seu efeito contra certas fraturas ósseas, especialmente coxofemorais, pois há estudos mostrando que mulheres que amamentam apresentam menos osteoporose e menos fraturas 40 .

Muitos trabalhos foram publicados mostrando como a amamentação se relaciona à amenorréia pós-parto e ao conseqüente maior espaçamento intergestacional. Outros benefícios para a mulher que amamenta são o retorno ao peso pré-gestacional mais precocemente e o menor sangramento uterino pós-parto (conseqüentemente, menos anemia), devido à involução uterina mais rápida provocada pela maior liberação de ocitocina, que é estimulada pela sucção precoce do bebê 40 .

A relação entre duração da amamentação e diminuição do peso pós-parto foi demonstrada em estudo brasileiro com 405 mulheres, em que a cada mês a mais de amamentação houve uma média de redução de $0,44 \mathrm{~kg}$ no peso da mãe 46 . 


\section{Evidências de algumas ações pró- amamentação que funcionam}

Estudos realizados em diferentes países consideram a Iniciativa Hospital Amigo da Criança uma ação extremamente efetiva, que leva ao incremento da prevalência e duração da amamentação exclusiva e total $26,47,48$. Na Suíça, o incremento nas taxas de amamentação que o país tem vivenciado desde 1994 deve-se em parte ao número crescente de Hospitais Amigos da Criança. Os serviços utilizam o título de Amigo da Criança como forma de se promover e isto tem influenciado as mulheres que desejam amamentar sobre a escolha do local para dar à luz 48 .

A avaliação sobre experiências dos países com a implementação das metas da Declaração de Innocenti, conduzida em 2002, mostrou que propostas como os Dez Passos são facilmente compreendidas e aceitas, porém sua sustentabilidade parece mais efetiva quando vinculada a uma abordagem que inclui política, legislação, reforma do sistema de saúde e intervenções na comunidade 49 . Os desafios para implementar a Iniciativa Hospital Amigo da Criança elencados por essa avaliação incluem: grande rotatividade de profissionais da saúde; estratégias para controle e manutenção do padrão de qualidade dos hospitais credenciados; sua inclusão no orçamento dos governos; adequado investimento no apoio à mãe após a alta da maternidade; clareza sobre como lidar com as mulheres HIV positivo; aperfeiçoamento da atenção à mulher durante o trabalho de parto e o parto; e integração com outras iniciativas em apoio às Metas do Milênio para o Desenvolvimento.

A sustentabilidade da Iniciativa Hospital Amigo da Criança foi avaliada no Brasil em 200250 e, na análise dos questionários de 137 hospitais amigos da criança, o que corresponde a $90 \%$ do total de 152 hospitais credenciados à época, observou-se que $92 \%$ cumpriram todos os dez passos. Os passos 1, 3, 6, 7, 8 e 9 apresentaram mais de $98 \%$ de cumprimento. O passo cinco foi o menos cumprido. Comparando-se as regiões do país, observou-se que no Nordeste, no Sul e no Sudeste, $90 \%$ dos hospitais foram aprovados em todos os dez passos. Na Região Norte, apenas $50 \%$ dos hospitais os cumpriram integralmente.

Revisão sistemática, realizada com o objetivo de avaliar as evidências sobre programas efetivos para aumentar o número de mulheres que iniciam a amamentação, selecionou 59 estudos. Estes foram agrupados segundo os temas educação em saúde; iniciativas gerais do setor saúde; Iniciativa Hospital Amigo da Criança; capacitação de profissionais da saúde; programa de su- plementação nutricional; apoio social de profissionais da saúde; apoio de pares; campanhas em meios de comunicação e outras intervenções 51 . Atividade em grupos pequenos durante o pré-natal, educação face a face, apoio de pares antes e após o parto mostraram efetividade como intervenções isoladas. Pacotes de intervenção que incluem apoio de pares e/ou campanhas em meios de comunicação associados a mudanças estruturais do setor saúde e/ou atividades educativas também se mostraram efetivos. Com relação aos serviços de saúde, mostraram-se relevantes as mudanças estruturais nas práticas de promoção do aleitamento materno nos hospitais, sendo o alojamento conjunto uma ação-chave 51 .

A efetividade do aconselhamento e de outras intervenções para melhorar a prática da amamentação foi avaliada por meio de revisão sistemática que incluiu apenas experimentos randomizados controlados e estudos de coorte provenientes de países desenvolvidos 52 . A busca foi direcionada para estudos que envolvessem aconselhamento ou intervenção comportamental aplicados em consultórios ou hospitais, excluindo-se, portanto, aqueles de origem comunitária ou de grupos de apoio mãe a mãe. Após levantamento em cinco importantes bancos de dados, 1.048 resumos foram selecionados e, destes, restaram para análise 22 experimentos randomizados controlados, oito não-experimentos e cinco revisões sistemáticas. Dois estudos foram considerados de boa qualidade, 12 regulares e 16 ruins. Os resultados indicaram que, de maneira geral, as intervenções parecem apresentar maiores efeitos em populações com baixos índices de amamentação como ponto de partida e que contribuíram para a melhoria das taxas de início e de continuidade da amamentação, porém com pouco efeito sobre duração prolongada.

Importante estudo brasileiro foi publicado no Lancet sobre os efeitos de profissionais capacitados sobre as práticas de aleitamento materno de mulheres em quatro cidades de Pernambuco. Uma fase pré-intervenção incluiu 318 mulheres que foram acompanhadas durante seis meses. Na segunda etapa, um ensaio randomizado, acompanhou 350 mães que deram à luz em dois hospitais cujas equipes haviam sido capacitadas por meio do curso de 18 horas da Iniciativa Hospital Amigo da Criança. Metade dessas mulheres foi sorteada para receber visitas domiciliares de promoção e apoio da amamentação enquanto a outra metade não recebeu as visitas. Dessa maneira, pôde-se observar se profissionais capacitados nos hospitais ou profissionais capacitados mais visitas domiciliares tinham efeitos similares sobre as taxas de amamentação exclusiva. Os resultados mostraram que a capacitação das 
equipes dos hospitais levou a uma taxa de $70 \%$ de amamentação exclusiva intra-hospitalar. Entretanto, isso não se sustentou após a volta para casa, quando apenas $30 \%$ das crianças amamentavam exclusivamente aos dez dias de vida. Daí, os autores concluírem sobre a necessidade de combinar ações em prol da amamentação também em nível comunitário 53.

Outro estudo brasileiro avaliou uma política de promoção, proteção e apoio ao aleitamento materno desenvolvida em unidades básicas de saúde. Essa política conhecida como Iniciativa Unidade Básica Amiga da Amamentação, organizada em dez passos a serem implementados pelos serviços de saúde, foi lançada pela Secretaria Estadual de Saúde do Rio de Janeiro em 1999. Na avaliação realizada em 24 unidades básicas de saúde de nove municípios, 13 unidades apresentavam desempenho regular e 11 desempenho fraco 54 . Constatou-se que 47,9\% das mães já forneciam água, chá, suco ou outros alimentos a seus bebês no primeiro mês de vida. Contudo, a prevalência da amamentação exclusiva foi maior no bloco de unidades básicas de saúde com desempenho regular. Os autores concluem que a implementação desse tipo de iniciativa pode contribuir para o aumento da prevalência da amamentação exclusiva no país, além de possivelmente melhorar a relação custo/efetividade das ações de promoção do aleitamento materno.

Sobre a prática de distribuir brindes para as mães ao deixar a maternidade são citados cinco estudos 52 , entre os quais uma revisão sistemática de nove experimentos randomizados cuja conclusão foi que o recebimento de brindes contendo amostras ou cupons de fórmula infantil leva à redução da prática de amamentação exclusiva. Por isso é de fundamental relevância implementar as recomendações do Código Internacional, das Resoluções subseqüentes e das legislações nacionais de proteção do aleitamento materno.

A influência da promoção comercial sobre as práticas de alimentação infantil e suas conseqüências sobre o desmame precoce, a desnutrição e a mortalidade infantil foram bastante discutidas nas décadas de 1960 e 1970. Em conseqüência disso, a OMS e o UNICEF realizaram, em 1979, a Reunião Conjunta sobre Alimentação do Lactente e da Criança Pequena em Genebra, Suíça 55. Ao final da reunião, foi recomendada a criação de um conjunto de normas fundamentadas em princípios éticos para nortear a promoção comercial de substitutos do leite materno: o Código Internacional de Comercialização de Substitutos do Leite Materno, que foi elaborado e aprovado em 1981 pela Assembléia Mundial da Saúde 56 .

Até 2005, 64 países haviam adotado medidas para implementar o código, dentre eles o Brasil, que o adotou como norma em 1988, abrangendo praticamente todas as suas disposições ${ }^{57}$. Uma análise dos avanços do nosso código - a Norma Brasileira de Comercialização de Alimentos para Lactentes e Crianças de Primeira Infância, Bicos, Chupetas e Mamadeiras (NBCAL) - foi realizada em 200658.

A implementação da NBCAL tem sido analisada como um empreendimento bem sucedido no Brasil em comparação com outros países, talvez por uma combinação de legislação abrangente, monitoramento regular e participação de grupos organizados 57. A International Baby Food Action Network (IBFAN), uma rede mundial de ativistas em defesa do direito de amamentar, criada em 1979 com a finalidade de acompanhar a implementação do código, desde então monitora as práticas de marketing relacionadas a produtos que competem com a amamentação. No Brasil, os resultados do monitoramento mais recente realizado em oito cidades de quatro estados, cujos dados foram coletados por pessoas capacitadas para a utilização de instrumentos padronizados 59, dão conta de que houve avanços porém persistem infrações à NBCAL na rotulagem dos produtos, na promoção comercial e nas informações disponíveis nas páginas eletrônicas.

\section{Comentário final}

O conjunto de estudos apresentados acima reforça a já difundida idéia na comunidade científica de que se acumulam as evidências sobre os benefícios da amamentação, tanto para a criança como para a mulher.

Verifica-se também o crescente interesse acerca da necessidade e das conseqüências do tipo de cuidado dispensado à criança no início da vida.

No entanto, estudos de impacto sobre como implementar essa prática são ainda escassos. Uma das razões pode ser a dificuldade de não se conseguir isolar e estudar um único fato (ou intervenção), devido à inter-relação de fatores ambientais e sócio-culturais que atuam na prática de amamentar, mesclando políticas públicas, benefícios, rotinas, ações de profissionais, apoio de pares etc. De toda maneira, permanece o desafio aos acadêmicos e profissionais de saúde pública, já que intervenções nesta área devem observar prioridades de custo e efetividade. 


\section{Resumo}

Este ensaio reúne uma seleção de estudos, particularmente revisões sistemáticas que têm contribuído para aumentar a compreensão sobre os benefícios do aleitamento materno para a criança e para a mulher e sua implementação. Realizou-se uma busca de artigos publicados a partir do ano 2000, sem, no entanto, deixar de lado estudos relevantes para o avanço do conhecimento publicados décadas atrás. Para a seleção dos estudos efetuou-se uma busca na Internet com base nas ferramentas disponíveis no PubMed e SciELO. Além dos aspectos para os quais há consenso, procurou-se incluir estudos sobre resultados controversos e outros que são instigantes, como os provenientes da neurobiologia. Verificam-se mudanças substanciais nas recomendações para políticas públicas em decorrência desses novos conhecimentos. Algumas investigações também têm sido realizadas com o objetivo de avaliar quais intervenções seriam mais efetivas para um aumento das práticas de amamentação. Procurou-se neste artigo dar destaque a: recomendações atuais sobre alimentação da criança pequena; importância da amamentação no início da vida; implicações do aleitamento materno para a saúde da criança; implicações do aleitamento materno para a saúde da mulher; e efetividade de algumas ações pró-amamentação.

Epidemiologia Nutricional; Aleitamento Materno; Leite Humano; Saúde da Mulher; Saúde da Criança

\section{Referências}

1. Popkin BM, Adair L, Akin JS, Black R, Briscoe J, Flieger W. Breast-feeding and diarrheal morbidity. Pediatrics 1990; 86:874-82.

2. Victora CG, Smith PG, Vaughan JP, Nobre LC, Lombardi C, Teixeira AM, et al. Evidence for protection by breast-feeding against infant deaths from infectious diseases in Brazil. Lancet 1987; 2:319-22.

3. Organização Mundial da Saúde. Estratégia global para a alimentação de lactentes e crianças de primeira infância. http://www.ibfan.org.br/ documentos (acessado em 11/Ago/2007).

4. Labbok MH, Wardlaw T, Blanc A, Clark D, Terreri $\mathrm{N}$. Trends in exclusive breastfeeding: findings from the 1990s. J Hum Lact 2006; 22:272-6.

5. Kramer MS, Kakuma R. The optimal duration of exclusive breastfeeding: a systematic review. Adv Exp Med Biol 2004; 554:63-77.

6. De Onis M, Victora CG. Gráficos de crescimento para bebês alimentados com leite materno. J Pediatr (Rio J) 2004; 80:85-7.

\section{Colaboradores}

T. S. Toma participou do desenho do artigo, da busca bibliográfica, da análise crítica da literatura selecionada, da redação do texto com ênfase em saúde da criança e intervenções, da revisão após recomendação dos pareceristas. M. F. Rea participou do desenho do artigo, da busca bibliográfica, da análise crítica da literatura selecionada, da redação do texto com ênfase em saúde da mulher e intervenções, da revisão após recomendação dos pareceristas.
7. De Onis M, Garza C, Onyango AW, Martorell R. WHO child growth standards. Acta Paediatr 2006; 95(450 Suppl):5-6.

8. Seal A, Kerac M. Operational implications of using 2006 World Health Organization growth standards in nutrition programmes: secondary data analysis. BMJ 2007; 334;733-8.

9. Bahl R, Frost C, Kirkwood BR, Edmond K, Martines J, Bhandari N, et al. Infant feeding patterns and risks of death and hospitalization in the first half of infancy: multicentre cohort study. Bull World Health Organ 2005; 83:418-26.

10. Organização Mundial da Saúde. Evidências científicas dos dez passos para o sucesso no aleitamento materno. Brasília: Organização Pan-Americana da Saúde; 2001. 
11. Dewey KG, Brown KH. Update on technical issues concerning complementary feeding of young children in developing countries and implications for intervention programs. Food Nutr Bull 2003; 24:528.

12. Edmond KM, Zandoh C, Quigley MA, AmengaEtego S, Owusu-Agyei S, Kirkwood BR. Delayed breastfeeding initiation increases risk of neonatal mortality. Pediatrics 2006; 117:380-6.

13. Wight NE. Hypoglycemia in breastfed neonates. Breastfeed Med 2006; 1:253-62.

14. Penders J, Thijs C, Vink C, Stelma FF, Snijders B, Kummeling I, et al. Factors influencing the composition of the intestinal microbiota in early infancy. Pediatrics 2006; 118:511-21.

15. Newburg DS, Ruiz-Palacios GM, Morrow AL. Human milk glycans protect infants against enteric pathogens. Annu Rev Nutr 2005; 25:37-58.

16. Forster DA, McLachlan HL. Breastfeeding initiation and birth setting practices: a review of the literature. J Midwifery Womens Health 2007; 52:27380.

17. Anderson GC, Moore E, Hepworth J, Bergman N. Early skin-to-skin contact for mothers and their healthy newborn infants (Cochrane Review). In: The Cochrane Library, Issue 1, 2007. Oxford: Update Software.

18. Mercer JS, Erickson-Owens DA, Graves B, Haley MM. Evidence-based practices for the fetal to newborn transition. J Midwifery Womens Health 2007; 52:262-72.

19. Uvnas-Moberg K. Oxytocin may mediate the benefits of positive social interactions and emotions. Psychoneuroendocrinology 1998; 23:819-35.

20. Moriceau S, Sullivan RM. Neurobiology of infant attachment. Dev Psychobiol 2005; 47:230-42.

21. Britton JR, Britton HL, Gronwaldt V. Breastfeeding, sensitivity, and attachment. Pediatrics 2006; 118: e1436-43.

22. Bowlby J. Apego. Volume 1 da trilogia apego e perda. São Paulo: Editora Martins Fontes; 1984

23. Brum EHM, Schermann L. Vínculos iniciais e desenvolvimento infantil: abordagem teórica em situação de nascimento de risco. Ciênc Saúde Coletiva 2004; 9:457-67.

24. Victora C, Barros FC. A questão da sobrevivência infantil no mundo e sua relevância para as Américas. Cadernos ESP: Escola de Saúde Pública do Ceará 2005; 1(1). http://www.esp.ce.gov.br/images/ documentos/cad_esp_vol01_n01.pdf (acessado em 06/Ago/2007).

25. Darmstadt GL, Bhutta ZA, Cousens S, Adam T, Walker N, De Bernis L, et al. Evidence-based, costeffective intervention: how many newborn babies can we save? Lancet 2005; 365:977-88.

26. Kramer MS, Chalmers B, Hodnett ED, Sevkovskaya Z, Dzikovich I, Shapiro S, et al. Promotion of Breastfeeding Intervention Trial (Probit): a randomized trial in the Republic of Belarus. JAMA 2001; 285:413-20.

27. Feachem RG, Koblinsky MA. Interventions for the control of diarrhoeal diseases among young children: promotion of breast-feeding. Bull World Health Organ 1984; 62:271-91.
28. Huffman SL, Combest C. Role of breast-feeding in the prevention and treatment of diarrhoea. J Diarrhoeal Dis Res 1990; 8:68-81.

29. Jackson KM, Nazar AM. Breastfeeding, the immune response, and long-term health. J Am Osteopath Assoc 2006; 106:203-7.

30. Cesar JA, Victora CG, Barros FC, Santos IS, Flores JA. Impact of breast feeding on admission for pneumonia during postneonatal period in Brazil: nested case-control study. BMJ 1999; 318:1316-20.

31. Oddy WH, Sly PD, Klerk NH, Landau LI, Kendall GE, Holt PG, et al. Breast feeding and respiratory morbidity in infancy: a birth cohort study. Arch Dis Child 2003; 88:224-8.

32. Chatzimichael A, Tsalkidis A, Cassimos D, Gardikis S, Tripsianis G, Deftereos S, et al. The role of breastfeeding and passive smoking on the development of severe bronchiolitis in infants. Minerva Pediatr 2007; 59:199-206.

33. Kuiper S, Muris JW, Dompeling E, Kester AD, Wesseling G, Knottnerus JA, et al. Interactive effect of family history and environmental factors on respiratory tract-related morbidity in infancy. J Allergy Clin Immunol 2007; 120:388-95.

34. Mihrshahi S, Ampon R, Webb K, Almqvist C, Kemp AS, Hector D, et al. The association between infant feeding practices and subsequent atopy among children with a family history of asthma. Clin Exp Allergy 2007; 37:671-9.

35. Mandhane PJ, Greene JM, Sears MR. Interactions between breast-feeding, specific parental atopy, and sex on development of asthma and atopy. J Allergy Clin Immunol 2007; 119:1359-66.

36. Sariachvili M, Droste J, Dom S, Wieringa M, Vellinga A, Hagendorens $M$, et al. Is breast feeding a risk factor for eczema during the first year of life? Pediatr Allergy Immunol 2007; 18:370-1.

37. Alm B, Wennergren G, Norvenius SG, Skjaerven R, Lagercrantz H, Helweg-Larsen K, et al. Breast feeding and the sudden infant death syndrome in Scandinavia, 1992-95. Arch Dis Child 2002; 86:400-2.

38. Marild S, Hansson S, Jodal U, Odén A, Svedberg K. Protective effect of breastfeeding against urinary tract infection. Acta Paediatr 2004; 93:164-8.

39. Horta BL, Bahl R, Martines JC, Victora CG. Evidence on the long-term effects of breastfeeding: systematic reviews and meta-analyses. Geneva: World Health Organization; 2007.

40. Rea MF. Os benefícios da amamentação para a saúde da mulher. J Pediatr (Rio J) 2004; 80(5 Suppl): S142-6.

41. Shema L, Ore L, Ben-Shachar M, Haj M, Linn S. The association between breastfeeding and breast cancer occurrence among Israeli Jewish women: a case control study. J Cancer Res Clin Oncol 2007; 133:539-46.

42. Kim Y, Choi JY, Lee KM, Park SK, Ahn SH, Noh DY, et al. Dose-dependent protective effect of breastfeeding against breast cancer among ever-lactated women in Korea. Eur J Cancer Prev 2007; 16:124-9.

43. Collaborative Group on Hormonal Factors in Breast Cancer. Breast cancer and breastfeeding: collaborative reanalysis of individual data from 47 epidemiological studies in 30 countries, including 50302 women with breast cancer and 96,973 women without the disease. Lancet 2002; 360:187-95. 
44. Martin RM, Middleton N, Gunnell D, Owen CG, Smith GD. Breastfeeding and cancer: the Boyd Orr cohort and a systematic review with meta-analysis. J Natl Cancer Inst 2005; 97:1446-57.

45. Okamura C, Tsubono Y, Ito K, Niikura H, Takano T, Nagase S, et al. Lactation and risk of endometrial cancer in Japan: a case-control study. Tohoku J Exp Med 2006; 208:109-15.

46. Kac G, Benicio MHD, Melendez GV, Valente JG, Struchiner CJ. Breastfeeding and postpartum weight retention in a cohort of Brazilian women. Am J Clin Nutr 2004; 79:487-93.

47. Lutter CK, Pérez-Escamilla R, Segall A, Sanghvi T, Teruya K, Wickham T. The effectiveness of a hospital-based program to promote exclusive breastfeeding among low-income women in Brazil. Am J Public Health 1997; 87:659-63.

48. Merten S, Dratva J, Ackermann-Liebrich U. Do baby-friendly hospitals influence breastfeeding duration on a national level? Pediatrics 2005; 116:702-8.

49. UNICEF Innocenti Research Centre. Celebrating the Innocenti Declaration on the Protection, Promotion and Support of Breastfeeding: past achievements, present challenges and the way forward for infant and young child feeding. Florence: UNICEF Innocenti Research Centre; 2005.

50. Araujo MFM, Otto AFN, Schmitz BAS. Primeira avaliação do cumprimento dos "Dez Passos para o Sucesso do Aleitamento Materno” nos Hospitais Amigos da Criança do Brasil. Rev Bras Saúde Matern Infant 2003; 3:411-9.

51. Fairbank L, O'Meara S, Renfrew MJ, Woolridge M, Sowden AJ, Lister-Sharp D. A systematic review to evaluate the effectiveness of interventions to promote the initiation of breastfeeding: executive summary. Health Technol Assess 2000; 4:1-171.
52. Guise JM, Palda V, Westhoff C, Chan BKS, Helfand $\mathrm{M}$, Lieu TA. The effectiveness of primary carebased interventions to promote breastfeeding: evidence review and meta-analysis for the U.S. Preventive Services Task Force. Ann Fam Med 2003; 1:70-80.

53. Coutinho SB, Lira PIC, Lima MC, Ashworth A. Comparison of the effect of two systems for the promotion of exclusive breastfeeding. Lancet 2005; 366:1094-100.

54. Oliveira MIC, Camacho LAB, Souza IEO. Promoção, proteção e apoio à amamentação na atenção primária à saúde no Estado do Rio de Janeiro, Brasil: uma política de saúde pública baseada em evidências. Cad Saúde Pública 2005; 21:1901-10.

55. Organização Mundial da Saúde/Fundo das Nações Unidas para a Infância. Reunião conjunta OMS/ UNICEF sobre a alimentação de lactentes e crianças pequenas: declaração e recomendações. Genebra: Organização Mundial da Saúde; 1979.

56. World Health Organization/The United Nations Children's Fund. The international code of marketing of breast-milk substitutes. Geneva: World Health Organization; 1981.

57. Sokol EJ. The Code handbook: a guide to implementing the International Code of Marketing of Breastmilk Substitutes. 2nd Ed. Den Haag: International Code Documentation Center; 2005.

58. Araujo MFM, Rea MF, Pinheira KA, Schmitz BAS. Avanços na Norma brasileira de comercialização de alimentos para idade infantil. Rev Saúde Pública 2006; 40:513-20.

59. De Divitiis R, Salve J, Toma TS. Monitoramento 2006: um resumo. http://www.ibfan.org.br/ monitoramento (acessado em 15/Ago/2007).

Recebido em 21/Ago/2007

Versão final reapresentada em 12/Mai/2008 Aprovado em 14/Mai/2008 